\title{
Stage IVA Bladder Cancer AJCC v8
}

National Cancer Institute

\section{Source}

National Cancer Institute. Stage IVA Bladder Cancer A/CC v8. NCI Thesaurus. Code C140425.

Stage IVA includes: (T 4b, N0, M0); (Any T, Any N, M1a). T4b: Extravesical tumor invades pelvic wall, abdominal wall. N0: No regional lymph node metastasis. MO: No distant metastasis. M1a: Distant metastasis limited to lymph nodes beyond the common iliacs. (AJCC 8th ed.) 